\title{
SY3-3
}

Symposium

\section{Non Adherence-the elephant in the room}

\section{Una Martin}

\section{University of Birmingham, $U K$}

Non-adherence to medication is common amongst hypertensive patients, with estimates ranging from 7-48\%. Total or partial non-adherence is thought to account for between 12-66\% of cases of apparent resistant hypertension. Measurement of patient adherence has historically been very challenging, and as a result, suboptimal adherence to a prescribed drug regimen often goes unrecognised in everyday clinical practice. Detecting non-adherence can be challenging and traditional methods such as direct questioning and pill counts are known to provide unreliable estimates of true medication adherence. Patients tend to underreport non-adherence and may take medication immediately before clinic appointments, so-called 'white coat adherence'. Conversely, clinical judgement alone is believed to overestimate the rate of non-adherence to antihypertensive medication.

The most commonly used approach in routine clinical practice is directly observed dosing. Blood pressure is measured before and after medication in a clinic setting with the patient observed as they take their tablets. Where a patient's blood pressure falls below $140 / 90 \mathrm{mmHg}$ following directly observed dosing, non-adherence to medication can be assumed. Electronic monitoring methods such as the medication event monitoring system (MEMS; AARDEX Group, Ltd, Sion, Switzerland) have been widely used to monitor adherence in clinical trials, because of their automaticity and precision of timing when patients take or omit doses.

The 'gold-standard' measure of medication adherence is to take a urine sample after the patient has taken their medications and examine the sample for relevant drug metabolites using high- performance liquid chromatography-mass spectrometry. This technique has been recently developed in the UK and Germany and has been shown to be effective at accurately detecting non-adherence to specific drugs types. It is increasingly being used in clinical practice in the UK although its availability is not yet widespread. 